\title{
Impact of allogeneic blood transfusions on clinical outcomes in severely burned patients
}

\author{
Kaserer, Alexander ; Rössler, Julian ; Slankamenac, Ksenija ; Arvanitakis, Michael ; Spahn, Donat R ;
} Giovanoli, Pietro ; Steiger, Peter ; Plock, Jan A

\begin{abstract}
BACKGROUND: Allogeneic blood transfusions are common in the treatment of severely burned patients as surgery may lead to major blood loss. However, transfusions are associated with a number of adverse events. Therefore, the purpose of our study was to investigate the impact of allogeneic blood transfusions on clinical outcomes in severely burned patients. METHODS: This retrospective study included all adult patients admitted to the burn center of the University Hospital Zurich between January 2004 and December 2014, with burn injuries greater than 10\% of total body surface area and receiving both surgical and intensive care treatment. Primary Endpoints were infectious or thromboembolic complications and mortality and secondary endpoints were length of hospital and ICU stay. Simple and multivariable logistic and linear regression models, adjusted for injury severity and confounders, were applied. RESULTS: 413 patients met inclusion criteria of which 212 patients (51\%) received allogenic blood products. After adjustment for injury severity and confounders, red blood cell transfusion was independently associated with wound infection (OR 13.5, 95\% CI 1.7-107, p = 0.014), sepsis (OR 8.3, 4.2-16.3; p < 0.001), pneumonia (OR 4.7, 2.2-10.0; p < 0.001), thrombosis (OR 3.0, 1.2-7.4; p = 0.015), central line infection (OR 34.7, 4.6-260; $\mathrm{p}=0.001$ ) and a longer ICU and hospital stay (difference 17.7, CI 12.1-23.4, $\mathrm{p}<0.001$ and 22.0, 15.8-28.2, $\mathrm{p}<0.001$, respectively). Fresh frozen plasma transfusion was independently associated with a longer ICU and hospital stay (difference 13.7, 95\% CI 5.5-21.8, p = 0.001 and $13.5,4.6-22.5, \mathrm{p}=0.003$, respectively). Platelet transfusion was independently associated with systemic inflammatory response syndrome (OR 4.5, 1.3-15.5; $\mathrm{p}=0.018$ ) and mortality (OR 5.8, 2.1-16.0; $\mathrm{p}=0.001)$. CONCLUSION: Transfusion of allogeneic blood products is associated with an increased infection rate and thromboembolic morbidity and a longer hospital stay in severely burned patients.
\end{abstract}

DOI: https://doi.org/10.1016/j.burns.2019.11.005

Posted at the Zurich Open Repository and Archive, University of Zurich

ZORA URL: https://doi.org/10.5167/uzh-180759

Journal Article

Accepted Version

Originally published at:

Kaserer, Alexander; Rössler, Julian; Slankamenac, Ksenija; Arvanitakis, Michael; Spahn, Donat R; Giovanoli, Pietro; Steiger, Peter; Plock, Jan A (2020). Impact of allogeneic blood transfusions on clinical outcomes in severely burned patients. Burns, 46(5):1083-1090.

DOI: https://doi.org/10.1016/j.burns.2019.11.005 
1 Impact of allogeneic blood transfusions on clinical outcomes in severely

3 Alexander Kaserer, M.D. ${ }^{1}$; Julian Rössler ${ }^{1}$; Ksenija Slankamenac, M.D., PhD²; Michael 4 Arvanitakis, M.D. ${ }^{3}$, Donat R. Spahn, M.D., F.R.C.A., Professor ${ }^{1}$; Pietro Giovanoli, M.D., 5 Professor $^{3}$; Peter Steiger, M.D. ${ }^{4}$; Jan Plock, M.D., Professor ${ }^{3}$;

6

$7 \quad{ }^{1}$ Institute of Anesthesiology, University and University Hospital Zurich, Zurich, Switzerland

$8 \quad 2$ Emergency Department, University Hospital Zurich, Zurich, Switzerland

$9{ }^{3}$ Department of Plastic Surgery and Hand Surgery, Burn Center, University and University 10 Hospital Zurich, Zurich, Switzerland

$11{ }^{4}$ Institute for Intensive Care Medicine, University Hospital Zurich, Zurich, Switzerland 12

13 Short title: Transfusions in burn patients

16 Corresponding Author:

17 Jan Plock, M.D., Professor

18 Department of Plastic Surgery and Hand Surgery, Burn Center, University and University

19 Hospital Zurich, Raemistrasse 100, CH-8091 Zurich, Switzerland;

20 Tel: +41(0) 442552738

21 E-mail: jan.plock@,usz.ch 


\section{Abstract}

Background: Allogeneic blood transfusions are common in the treatment of severely burned patients as surgery may lead to major blood loss. However, transfusions are associated with a number of adverse events. Therefore, the purpose of our study was to investigate the impact of allogeneic blood transfusions on clinical outcomes in severely burned patients.

Methods: This retrospective study included all adult patients admitted to the burn center of the University Hospital Zurich between January 2004 and December 2014, with burn injuries greater than $10 \%$ of total body surface area and requiring both surgical and intensive care treatment. Primary Endpoints were infectious or thromboembolic complications and mortality and secondary endpoints were length of hospital and ICU stay. Simple and multivariable logistic and linear regression models, adjusted for injury severity and confounders, were applied.

Results: 413 patients met inclusion criteria of which 212 patients (51\%) required allogenic blood products. After adjustment for injury severity and confounders, red blood cell transfusion was independently associated with wound infection (OR $13.5,95 \%$ CI 1.7 to $107, p=0.014$ ), sepsis (OR 8.3, 4.2 to 16.3; $\mathrm{p}<0.001$ ), pneumonia (OR 4.7, 2.2 to $10.0 ; \mathrm{p}<0.001$ ), thrombosis (OR 3.0, 1.2 to $7.4 ; \mathrm{p}=0.015)$, central line infection (OR 34.7, 4.6 to $260 ; \mathrm{p}=0.001$ ) and a longer ICU and hospital stay (difference $17.7,95 \%$ CI 12.1 to $23.4, \mathrm{p}<0.001$ and $22.0,15.8$ to 28.2 , $\mathrm{p}<0.001$, respectively). Fresh frozen plasma transfusion was independently associated with a longer ICU and hospital stay (difference $13.7,95 \%$ CI 5.5 to $21.8, p=0.001$ and $13.5,4.6$ to $22.5, p=0.003$, respectively). Platelet transfusion was independently associated with systemic inflammatory response syndrome (OR 4.5, 1.3 to $15.5 ; \mathrm{p}=0.018)$ and mortality (OR 5.8, 2.1 to $16.0 ; \mathrm{p}=0.001)$. 
50 Conclusion: Transfusion of allogeneic blood products is associated with an increased infection 51 rate and thromboembolic morbidity and a longer hospital stay in severely burned patients.

52

53 Key words: Burn; blood products; infections; morbidity; mortality; thromboembolic events; 54

55 


\section{Background}

Transfusion and coagulation management in severely burned patients is challenging. Thermal injuries with extended soft tissue injury and subsequent inflammatory response lead to diffuse activation of coagulation factors culminating in a hypercoagulable state.[1,2] While this alone increases the risk of developing venous thromboembolism, patients suffering from burn injuries are further exposed to numerous additional risk factors for infections, like repetitive surgical procedures, use of intravascular catheters, prolonged immobilization and multiple blood transfusions.[3, 4] Transfusions are common in burn surgery, as tangential excision may lead to major blood loss.[5] Here, apart from balanced volume replacement, hemostatic resuscitation traditionally meant early transfusion of packed red blood cells (RBC), fresh frozen plasma (FFP) and platelet concentrate (PLT) at a fixed ratio.[6, 7] However, allogenic blood transfusions are associated with a number of adverse events and it was shown that their reduction improves clinical outcomes including mortality.[4, 8] A factor-based coagulation management guided by viscoelastic point of care tests[9] has recently been proposed as a new approach of hemostatic resuscitation leading to a decreased transfusion requirement with beneficial outcomes in trauma patients.[10-13] However, burn victims are not taken specifically into account. Identification of adverse effects of allogeneic blood transfusions in severely burned patients may further promote the implementation of such goal directed coagulation management in burn patients in order to reduce transfusion requirements.[14] Therefore, the purpose of our retrospective study was to investigate the impact of allogeneic blood transfusions on clinical outcomes in severely burned patients.

\section{Methods}

\section{$\underline{\text { Study setting and patient selection }}$}

This study was approved by the responsible ethics committee of the canton of Zurich (KEKZH-Nr.: 2015-111). Data handling was in accordance with the Good Clinical Practice 
Guidelines and the Declaration of Helsinki for biomedical research. The study included all adult patients admitted to the burn center of the University Hospital Zurich between January 2004 and December 2014 with burn injuries greater than $10 \%$ of total body surface area (TBSA) and requiring both surgical and intensive care treatment. No substantial changes to the transfusion and coagulation algorithm were made during the observed period. To prevent a possible bias of our results we purposely terminated the observation period at the end of 2014 as in 2015 a new transfusion and coagulation algorithm was introduced at our burn center. Exclusion criteria were incomplete medical records, conservatively treated superficial burns (grade I-IIa), secondary admissions with older injuries (after the acute phase treatment in regional hospital or abroad) and all patients who received palliation-focused care because of terminal injuries. Figure 1 presents a flowchart of the patient selection.

\section{Data collection}

From the internal electronic database of the University Hospital Zurich, we extracted patient's demographics, abbreviated burn severity index (ABSI) score[15], TBSA, co-morbidities, additional injuries including inhalation injury, administered RBC, PLT and FFP transfusions, thromboembolic events, systemic inflammatory response syndrome (SIRS), sepsis, pneumonia, central line infections, urinary tract infections, wound infections, length of ICU and hospital stay as well as mortality. Additionally, we reviewed anesthesia records for administered blood products and clotting factors during all surgical procedures. Extracted data was entered into a spreadsheet (Excel, Microsoft Office 2016, Redmond, WA, United States) and scores and values were calculated. Comorbidities were summarized calculating the Charlson co-morbidity index.[16] All patients received venous thromboembolism prophylaxis with low molecular weight heparin. SIRS and sepsis were defined according to the definition consensus of the American Burn Association related to the observation period.[17] The ABSI is a model to assess accurately[15] probability of mortality, that uses age, TBSA, inhalational injury, gender 
and the presence of full thickness burns to generate a score. It is therefore also used as an equivalent of burn injury severity.

\section{Endpoints and outcome variables}

The aim of our study was to analyze the impact of allogeneic blood transfusions on clinical outcomes. Primary endpoints were thromboembolic events, SIRS, sepsis, pneumonia, central line infection, urinary tract infection, wound infections and mortality. Secondary endpoints were length of ICU and hospital stay in severely burned patients.

\section{Statistical analyses}

In a first step of the analysis, we expressed distribution of variables using means and standard deviation (SD) for normally distributed continuous data, and medians and interquartile ranges (IQR) for non-normally distributed data. We tested data for normality with the KolmogorowSmirnow test and performed quantile-quantile plots of dependent variables. In a second step, we used simple logistic and linear regression models, followed by the main analysis, a multivariable logistic and linear regression model for the following parameters: thrombosis, SIRS, sepsis, pneumonia, central line-associated infections, urinary tract infections, wound infection, mortality, length of ICU stay and length of hospital stay. Thereby we adjusted for the following confounders known from the literature, based on the clinical experience and statistical models: ABSI-score, Charlson co-morbidity index, the presence of additional injuries and the administration of clotting factors.

For all results, we reported point estimates, $95 \%$ confidence intervals $(\mathrm{CI})$ and $p$-values $\leq 0.05$ considered significant. We performed the statistical analyses using the statistical program STATA SE (version 14, Stata Corp., College Station, Texas, United States). 


\section{Results}

We retrospectively screened 810 patients admitted to the burn ICU of the University Hospital of Zurich during a 10-year period from 2004 to 2104 . We then excluded 397 patients who did not meet inclusion criteria. Of the remaining 413 patients, 212 patients (51\%) required allogenic blood products, while the other 201 patients (49\%) did not (Figure 1).

The two groups were well balanced in size with a similar mean age. The prevalence of comorbidities was comparable with a Charlson co-morbidity index $<4$ in $91.5 \%$ of patients who required transfusions and in $94.0 \%$ of patients who did not. Patients requiring allogenic blood transfusions were female in $32.5 \%$ compared to $18.9 \%$ in non-transfused patients. Further group differences were found regarding the severity of the burn injury, where patients receiving allogenic blood products had a larger burned TBSA and a higher ABSI score (6 \pm 2 vs. $8 \pm 2$ ), as well as more inhalation or other additional injuries (Table 1). These group differences were considered as confounders in our regression model.

Among patients requiring RBC transfusions, sepsis occurred in nearly half of all patients. Other frequently observed complications were pneumonia (25\%), central line infections $(17 \%)$ and thrombosis (12.7\%). Table 2 gives a detailed overview on the incidence of observed complications. After adjusting for confounders, RBC transfusion was independently associated with central line infections, wound infections, sepsis, pneumonia and thrombosis. Moreover, RBC transfusion was independently associated with a longer ICU and hospital stay. Unadjusted and adjusted odds ratios for each outcome are presented in Table 2. Mortality was not affected by RBC transfusions after adjustment (Table 2, Figure 2).

FFP was administered in 51 out of 413 included patients (12\%). Leading complications in patients requiring FFP transfusions were sepsis $(60.8 \%)$, pneumonia $(33.3 \%)$ and central line infections (23.5\%). Incidence of all observed complications as well as unadjusted and adjusted odds ratio for each outcome is presented in Table 3 and Figure 2. After adjustment, FFP 
transfusion was independently associated with a longer ICU and hospital stay only. No significant association with other outcomes, in particular mortality, was observed (Table 3).

PLT transfusions were administered in 31 patients out of 413 included patients $(7.5 \%)$.

Frequently observed complications in patients requiring PLT transfusion were sepsis $(74.2 \%)$, pneumonia (41.9\%), thrombosis $(22.6 \%)$ and central line infections $(22.6 \%)$. Mortality was high in patients requiring PLT transfusion (58.1\%). Table 4 presents unadjusted and adjusted odds ratios in detail. After adjustment, PLT transfusion was independently associated with SIRS and mortality, but not with sepsis or other outcomes (Table 4, Figure 2).

\section{Discussion}

Allogeneic blood transfusions are common in the treatment of severely burned patients as surgery may lead to major blood loss.[5, 18, 19] Currently, fixed ratio transfusion of RBC, PLT and FFP is still widely used and recommended for hemostatic resuscitation.[6, 7] However, transfusions may additionally lead to complications and worse clinical outcomes.[4, 8] In this study, we retrospectively analyzed the data of 413 burn patients admitted to the largest burn center in Switzerland. We calculated multiple regression models adjusting for possible confounders and found that RBC transfusion was associated with central line infections, wound infections, sepsis, pneumonia, thrombosis and a longer ICU and hospital stay; FFP transfusion was associated with a longer ICU and hospital stay and PLT transfusion was independently associated with SIRS and mortality.

Allogeneic blood transfusion is associated to several adverse effects.[4, 8] Repetitive surgeries with major blood loss and a concomitant hypercoagulability of burn patients complicates hemostatic and transfusion management.[1,2,7] As a result of the overwhelming response to severe thermal injury burn patients have increased levels of proinflammatory cytokines, which blunt erythropoietic response in the bone marrow.[20] The distinction between acute blood loss 
anemia and anemia due to bone marrow dysfunction in burn patients is therefore an essential framework to lower the incidence of anemia and reduce transfusion requirement. For nonburned patients a multimodal treatment concept was introduced to reduce allogeneic blood transfusions. Such Patient Blood Management programs were confirmed to improve outcomes and reduce costs.[8, 21, 22] However, burn patients are not specially taken into account by such programs and physicians are still treating severely burned patients with an ample of transfusions reaching an overall transfusion rate up to $97.7 \%$.[7, 18] The adverse effect of such an overflowing transfusion management cannot be neglected. Palmieri et al. showed that a restrictive transfusion strategy is feasible and well tolerated in burned patients.[23] Moreover, her group showed that a restrictive transfusion strategy halved the administered blood products without worsening outcomes in major burns.[24] In another prospective randomized trial of 345 patients Palmieri et al. demonstrated, that a restrictive transfusion strategy is not only well tolerated, but also beneficial by reducing length of ventilatory support and ICU stay.[25] While these trials were conducted in multiple centers in the United States, this conclusion is in line with our findings showing an increased ICU and hospital stay in patients requiring blood transfusions in a single European center. This implies a generalizability of the underlying results regarding the impact of transfusions on clinical outcomes.

Preventing infections is important in the treatment of burn patients. The burned body area is missing its natural barrier function and patients treated with allogeneic blood products are further exposed to a transfusion-related immunomodulation effect, as well as changes that may occur with old blood.[26] Moreover, a restrictive management in the antibiotic therapy should prevent the development of multi-resistant germs. These factors favor the occurrence of infections in burn patients, which eventually may lead to sepsis. Our findings reflect this fact by showing an independent association of RBC transfusion with central line infections, wound infections, pneumonia and sepsis confirming the findings of Palmieri et al.[27] 
In line with our results, transfusion was detected as a possible risk factor for thrombosis in burned patients. $[3,28]$ Recently, an association of perioperative RBC transfusion with venous

211 thromboembolism was confirmed in 750.937 non-burned patients undergoing surgery.[29]

212 Burned patients additionally suffer from extended soft tissue injury and subsequent

213 inflammatory response leading to a diffuse activation of coagulation factors, which culminates

214 in a hypercoagulable state.[1,2] While this alone increases the risk of developing venous thromboembolism, burn patients are further exposed to numerous additional risk factors, like the need of central lines or other intravascular catheters and prolonged immobilization.

217 Glance et al. showed an association of intraoperative blood transfusion with a higher risk of 218 mortality in anemic patients undergoing noncardiac surgery.[4] But also in burn patients 219 Palmieri et al. and Tavousi et al. found an increased mortality due to blood transfusions.[27, 30] However, in our analysis only PLT transfusion was independently associated with mortality, but not RBC or FFP transfusion. PLT transfusion is not frequently necessary in

222 burned patients. Platelets drop below a critical threshold predominantly in acute situations such as major bleeding requiring successive massive transfusion. Therefore, we interpret the association of PLT transfusion with mortality more as an expression of a deranging situation, rather than a causation.

\section{Limitations}

This study has some limitations. Importantly it was a retrospective study and we are limited by the present methodological confines, as a retrospective analysis can only indicate a possible association and not causation. Further, as for all post-hoc studies, a power analysis would be inappropriate, however the confidence intervals of certain events in the regression model suggest an inadequate sample size. Due to overlapping transfusion requirements for different blood products, none of our patients had FFP or PLT transfusion only. This might mask the

234 isolated effect of each blood product partly. Blood products are known to be transfused in higher 
235 amounts in sicker patients. Thereby, any retrospective analysis about blood and outcome is 236 challenging. Although patients requiring blood transfusions had a larger TBSA and more 237 concomitant inhalation injuries, we are confident that our approach is sufficient to capture 238 possible negative impact of allogenic blood products. We adjusted our analysis for the ABSI 239 score, which was recently proven for its accuracy.[15] Additionally, with the adjustment for the 240 Charlson co-morbidity index, the presence of additional injuries and the administration of 241 clotting factors we eliminated other possible confounders in order to focus on the impact of 242 transfusions on our outcomes. However, there might be some confounders for which we cannot 243 account and correct retrospectively. Additional confounding factors known for blood products 244 (e.g., elderly, cardiovascular comorbidities, anemia) can reasonably be excluded as this 245 analyzed group of patients is in general a young and healthy population without comorbidities 246 (mean age of 43-45 years). Therefore, we are convinced that our data are robust and conclusive.

\section{Conclusion}

249 Transfusion of allogeneic blood products is associated with an increased morbidity in severely 250 burned patients. Our findings therefore support the approach of a restrictive transfusion strategy 251 in severely burned patients. 


\section{Conflicts of interests:}

254

255

AK, JR, KS, MA, PG, PS and JAP have no conflicts of interests to declare.

Dr. Spahn's academic department is receiving grant support from the Swiss National Science Foundation, Berne, Switzerland, the Swiss Society of Anesthesiology and Reanimation (SGAR), Berne, Switzerland, the Swiss Foundation for Anesthesia Research, Zurich, Switzerland, Vifor SA, Villars-sur-Glâne, Switzerland.

Dr. Spahn is co-chair of the ABC-Trauma Faculty, sponsored by unrestricted educational grants from Novo Nordisk Health Care AG, Zurich, Switzerland, CSL Behring GmbH, Marburg, Germany, LFB Biomédicaments, Courtaboeuf Cedex, France and Octapharma AG, Lachen, Switzerland.

Dr. Spahn received honoraria / travel support for consulting or lecturing from:

Danube University of Krems, Austria, US Department of Defense, Washington, USA, European Society of Anesthesiology, Brussels, BE, Korean Society for Patient Blood Management, Seoul, Korea, Korean Society of Anesthesiologists, Seoul, Korea, Baxter / Baxalta AG, Volketswil, Switzerland, Bayer AG, Zürich, Switzerland, Bayer Pharma AG, Berlin, Germany, B. Braun Melsungen AG, Melsungen, Germany, Boehringer Ingelheim GmbH, Basel, Switzerland, Bristol-Myers-Squibb, Rueil-Malmaison Cedex, France and Baar, Switzerland, CSL Behring GmbH, Hattersheim am Main, Germany and Berne, Switzerland, Celgene International II Sàrl, Couvet, Switzerland, Daiichi Sankyo AG, Thalwil, Switzerland, Haemonetics, Braintree, MA, USA, Instrumentation Laboratory (Werfen), Bedford, MA, USA, LFB Biomédicaments, Courtaboeuf Cedex, France, Merck Sharp \& Dohme, Kenilworth, New Jersey, USA, Octapharma AG, Lachen, Switzerland, Paion UK Ltd, Cambridge, UK and Deutschland GmbH, Aachen, Germany, Pharmacosmos A/S, Holbaek, Denmark, Photonics Healthcare B.V., Utrecht, Netherlands, Pierre Fabre Pharma, Alschwil, Switzerland, Roche Diagnostics International Ltd, Reinach, Switzerland, Sarstedt AG \& Co., Sevelen, Switzerland and Nümbrecht, Germany, Tem International GmbH, Munich, Germany, Vifor Pharma, 
279 Munich, Germany, Vienna, Austria, Paris, France and Villars-sur-Glâne, Switzerland, Vifor 280 (International) AG, St. Gallen, Switzerland.

281

\section{Authors' Contributions}

283 AK and JR did the artwork, contributed to data interpretation, drafting and critical revision of 284 the manuscript. KS contributed to statistical analysis, data interpretation and critical revision 285 of the manuscript. MA contributed to data collection, data interpretation and critical revision 286 of the manuscript. DS, PG and PS contributed to data interpretation and critical revision of the 287 manuscript. JP designed and coordinated the study, provided the data, contributed to data 288 interpretation and critical revision of the manuscript. All authors read and approved the final 289 manuscript.

290

291 Funding: This research did not receive any specific grant from funding agencies in the public, 292 commercial, or not-for-profit sectors. 


\section{References}

295 [1] Barret JP, Dziewulski PG. Complications of the hypercoagulable status in burn injury. 296 Burns. 2006;32:1005-8.

297

298

299

300

301

302

303

304 305

[2] Meizoso JP, Ray JJ, Allen CJ, Van Haren RM, Ruiz G, Namias N, et al. Hypercoagulability and venous thromboembolism in burn patients. Semin Thromb Hemost. 2015;41:43-8.

[3] Mullins F, Mian MA, Jenkins D, Brandigi C, Shaver JR, Friedman B, et al. Thromboembolic complications in burn patients and associated risk factors. J Burn Care Res. 2013;34:355-60.

[4] Glance LG, Dick AW, Mukamel DB, Fleming FJ, Zollo RA, Wissler R, et al. Association between intraoperative blood transfusion and mortality and morbidity in patients undergoing noncardiac surgery. Anesthesiology. 2011;114:283-92.

[5] Farny B, Fontaine M, Latarjet J, Poupelin JC, Voulliaume D, Ravat F. Estimation of blood loss during adult burn surgery. Burns. 2018;44:1496-501.

[6] Holcomb JB, Tilley BC, Baraniuk S, Fox EE, Wade CE, Podbielski JM, et al. Transfusion of plasma, platelets, and red blood cells in a 1:1:1 vs a 1:1:2 ratio and mortality in patients with severe trauma: the PROPPR randomized clinical trial. JAMA. 2015;313:471-82.

[7] Gurney JM, Kozar RA, Cancio LC. Plasma for burn shock resuscitation: is it time to go back to the future? Transfusion. 2019;59:1578-86.

[8] Leahy MF, Hofmann A, Towler S, Trentino KM, Burrows SA, Swain SG, et al. Improved outcomes and reduced costs associated with a health-system-wide patient blood management 
program: a retrospective observational study in four major adult tertiary-care hospitals. Transfusion. 2017;57:1347-58.

[9] Stein P, Kaserer A, Spahn GH, Spahn DR. Point-of-Care Coagulation Monitoring in Trauma Patients. Semin Thromb Hemost. 2017;43:367-74.

[10] Stein P, Kaserer A, Sprengel K, Wanner GA, Seifert B, Theusinger OM, et al. Change of 318 transfusion and treatment paradigm in major trauma patients. Anaesthesia. 2017;72:1317-26.

[11] Gonzalez E, Moore EE, Moore HB, Chapman MP, Chin TL, Ghasabyan A, et al. Goal320 directed Hemostatic Resuscitation of Trauma-induced Coagulopathy: A Pragmatic 321 Randomized Clinical Trial Comparing a Viscoelastic Assay to Conventional Coagulation 322 Assays. Ann Surg. 2016;263:1051-9.

323 [12] Gorlinger K, Dirkmann D, Hanke AA, Kamler M, Kottenberg E, Thielmann M, et al. First324 line therapy with coagulation factor concentrates combined with point-of-care coagulation 325 testing is associated with decreased allogeneic blood transfusion in cardiovascular surgery: a 326 retrospective, single-center cohort study. Anesthesiology. 2011;115:1179-91.

327 [13] Spahn DR, Bouillon B, Cerny V, Duranteau J, Filipescu D, Hunt BJ, et al. The European 328 guideline on management of major bleeding and coagulopathy following trauma: fifth edition. 329 Critical care. 2019;23:98.

330 [14] Welling H, Ostrowski SR, Stensballe J, Vestergaard MR, Partoft S, White J, et al. 331 Management of bleeding in major burn surgery. Burns. 2019;45:755-62. 

mortality. Ann Burns Fire Disasters. 2018;31:89-93.

334

335 336 $344 \quad 2018 ; 39: 286-94$.

345 [20] Posluszny JA, Jr., Gamelli RL. Anemia of thermal injury: combined acute blood loss 346 anemia and anemia of critical illness. J Burn Care Res. 2010;31:229-42.

347 [21] Mehra T, Seifert B, Bravo-Reiter S, Wanner G, Dutkowski P, Holubec T, et al. Implementation of a patient blood management monitoring and feedback program significantly

[16] Charlson ME, Pompei P, Ales KL, MacKenzie CR. A new method of classifying prognostic comorbidity in longitudinal studies: development and validation. J Chronic Dis. $1987 ; 40: 373-83$.

[17] Greenhalgh DG, Saffle JR, Holmes JHt, Gamelli RL, Palmieri TL, Horton JW, et al. American Burn Association consensus conference to define sepsis and infection in burns. $\mathbf{J}$ Burn Care Res. 2007;28:776-90.

[18] Wu G, Zhuang M, Fan X, Hong X, Wang K, Wang H, et al. Blood transfusions in severe burn patients: Epidemiology and predictive factors. Burns. 2016;42:1721-7.

[19] Hasan S, Mosier MJ, Conrad P, Szilagyi A, Gamelli RL, Muthumalaiappan K. Terminal Maturation of Orthochromatic Erythroblasts Is Impaired in Burn Patients. J Burn Care Res. reduces transfusions and costs. Transfusion. 2015;55:2807-15. 
351 Patient Blood Management Program Based on a Three-pillar Strategy: A Systematic Review 352 and Meta-analysis. Ann Surg. 2019;269:794-804.

353 [23] Palmieri TL. Burn injury and blood transfusion. Curr Opin Anaesthesiol. 2019;32:247-51.

354 [24] Palmieri TL, Holmes JHt, Arnoldo B, Peck M, Potenza B, Cochran A, et al. Transfusion 355 Requirement in Burn Care Evaluation (TRIBE): A Multicenter Randomized Prospective Trial 356 of Blood Transfusion in Major Burn Injury. Ann Surg. 2017;266:595-602.

357 [25] Palmieri TL, Holmes JH, Arnoldo B, Peck M, Cochran A, King BT, et al. Restrictive 358 Transfusion Strategy Is More Effective in Massive Burns: Results of the TRIBE Multicenter 359 Prospective Randomized Trial. Mil Med. 2019;184:11-5.

360 [26] Nielsen HJ, Reimert CM, Dybkjaer E, Roed J, Alsbjorn B. Bioactive substance 361 accumulation and septic complications in a burn trauma patient: effect of perioperative blood 362 transfusion? Burns. 1997;23:59-63.

363 [27] Palmieri TL, Caruso DM, Foster KN, Cairns BA, Peck MD, Gamelli RL, et al. Effect of 364 blood transfusion on outcome after major burn injury: a multicenter study. Crit Care Med. $365 \quad 2006 ; 34: 1602-7$

366 [28] Johnson DJ, Scott AV, Barodka VM, Park S, Wasey JO, Ness PM, et al. Morbidity and 367 Mortality after High-dose Transfusion. Anesthesiology. 2016;124:387-95. 

369 of Perioperative Red Blood Cell Transfusions With Venous Thromboembolism in a North 370 American Registry. JAMA Surg. 2018;153:826-33.

371 [30] Tavousi SH, Ahmadabadi A, Sedaghat A, Khadem-Rezaiyan M, Yaghoubi Moghaddam 372 Z, Behrouzian MJ, et al. Blood transfusion in burn patients: Triggers of transfusion in a referral 373 burn center in Iran. Transfus Clin Biol. 2018;25:58-62. 
Table 1: Patients' characteristic.

\begin{tabular}{|c|c|c|}
\hline & $\begin{array}{l}\text { Patients requiring NO } \\
\text { allogeneic blood transfusions } \\
(\mathrm{n}=\mathbf{2 0 1})\end{array}$ & $\begin{array}{l}\text { Patients requiring allogeneic } \\
\text { blood transfusions } \\
(\mathrm{n}=\mathbf{2 1 2})\end{array}$ \\
\hline Age, years, mean (SD) & $43.8(16.3)$ & $45.8(17.9)$ \\
\hline Sex female $(\%)$ & $38(18.9 \%)$ & $69(32.5 \%)$ \\
\hline $\begin{array}{l}\text { Charlson co-morbidity index, } \\
\text { median (IQR) }\end{array}$ & $0(0-1)$ & $0(0-2)$ \\
\hline$-\quad<4$ & $189(94.0 \%)$ & $194(91.5 \%)$ \\
\hline$-\quad \geq 4$ & $12(6.0 \%)$ & $18(8.5 \%)$ \\
\hline Burn \% TBSA, median (IQR) & $15(12-24)$ & $30(18-41)$ \\
\hline ABSI score, mean (SD) & $6(2)$ & $8(2)$ \\
\hline $3^{\text {rd }}$ Burn & $88(43.8 \%)$ & $163(76.9 \%)$ \\
\hline Inhalation injury & $21(10.4 \%)$ & $36(17.0 \%)$ \\
\hline $\begin{array}{l}\text { Additional injuries } \\
\text { (other than inhalation injury) }\end{array}$ & $3(1.5 \%)$ & $12(5.7 \%)$ \\
\hline
\end{tabular}


Table 2: Outcome Analysis for RBC transfusion in severely burned patients ( $\mathrm{n}=413)$.

\begin{tabular}{|c|c|c|c|c|c|c|}
\hline & $\begin{array}{c}\text { No RBC } \\
\text { transfusions } \\
\mathbf{n}=\mathbf{2 0 1} \\
\end{array}$ & $\begin{array}{c}\text { Requiring RBC } \\
\text { transfusions } \\
n=212\end{array}$ & unadjusted OR (95\% CI) & p-value & adjusted OR (95\% CI) & p-value \\
\hline Thrombosis & $8(4.0 \%)$ & $27(12.7 \%)$ & $3.5(1.6$ to 7.9$)$ & $\mathrm{p}=0.002$ & $3.0(1.2$ to 7.4$)$ & $\mathrm{p}=0.015$ \\
\hline SIRS & $4(2.0 \%)$ & $15(7.1 \%)$ & $3.8(1.2$ to 11.5$)$ & $\mathrm{p}=0.021$ & $2.1(0.6$ to 7.5$)$ & $\mathrm{p}=0.245$ \\
\hline Sepsis & $13(6.5 \%)$ & $103(48.6 \%)$ & $13.7(7.3$ to 25.5$)$ & $\mathrm{p}<0.001$ & $8.3(4.2$ to 16.3$)$ & $\mathrm{p}<0.001$ \\
\hline Pneumonia & $10(5.0 \%)$ & $53(25 \%)$ & $6.4(3.1$ to 12.9$)$ & $\mathrm{p}<0.001$ & $4.7(2.2$ to 10.0$)$ & $\mathrm{p}<0.001$ \\
\hline Central line-associated infections & $1(0.5 \%)$ & $36(17.0 \%)$ & $40.9(5.6$ to 301$)$ & $\mathrm{p}<0.001$ & $34.7(4.6$ to 260$)$ & $\mathrm{p}=0.001$ \\
\hline Urinary tract infections & $4(2.0 \%)$ & $14(6.6 \%)$ & $3.5(1.1$ to 10.8$)$ & $\mathrm{p}=0.030$ & $2.6(0.7$ to 9.0$)$ & $\mathrm{p}=0.138$ \\
\hline Wound infection & $1(0.5 \%)$ & $19(9.0 \%)$ & 19.7 (2.6 to 149$)$ & $\mathrm{p}=0.004$ & $13.5(1.7$ to 107$)$ & $\mathrm{p}=0.014$ \\
\hline \multirow[t]{2}{*}{ Mortality } & $19(9.5 \%)$ & $44(20.8 \%)$ & $2.5(1.4$ to 4.5$)$ & $\mathrm{p}=0.002$ & $0.8(0.4$ to 1.7$)$ & $\mathrm{p}=0.504$ \\
\hline & & & $\begin{array}{c}\text { unadjusted difference } \\
(95 \% \mathrm{CI})\end{array}$ & & $\begin{array}{c}\text { adjusted difference } \\
(95 \% \mathrm{CI})\end{array}$ & \\
\hline Length of ICU stay (days) & $7(3-12)$ & $28(13.5-43)$ & $26.1(20.9$ to 31.2$)$ & $\mathrm{p}<0.001$ & $17.7(12.1$ to 23.4$)$ & $\mathrm{p}<0.001$ \\
\hline Length of hospital stay (days) & $15(11-22)$ & $36(21.5-58)$ & $27.9(22.4$ to 33.4$)$ & $\mathrm{p}<0.001$ & $22.0(15.8$ to 28.2$)$ & $\mathrm{p}<0.001$ \\
\hline
\end{tabular}

Adjusted for the following confounders: ABSI-score, Charlson co-morbidity index, the presence of additional injuries and administration of clotting factors. All parametric results were presented as median (interquartile range). $\mathrm{OR}=$ odds ratio; $\mathrm{CI}=$ confidence interval; SIRS = Systemic Inflammatory Response Syndrome; 
Table 3: Outcome analysis for FFP transfusions in severely burned patients $(n=413)$.

\begin{tabular}{|c|c|c|c|c|c|c|}
\hline & $\begin{array}{c}\text { No FFP } \\
\text { transfusions } \\
n=362\end{array}$ & $\begin{array}{c}\begin{array}{c}\text { Requiring FFP } \\
\text { transfusions } \\
n=51\end{array} \\
\end{array}$ & unadjusted OR (95\% CI) & p-value & adjusted OR (95\% CI) & p-value \\
\hline Thrombosis & $30(8.3 \%)$ & $5(9.8 \%)$ & $1.2(0.4$ to 3.3$)$ & $\mathrm{p}=0.72$ & $0.6(0.2-1.6)$ & $\mathrm{p}=0.303$ \\
\hline SIRS & $14(3.9 \%)$ & $5(9.8 \%)$ & $2.7(0.9$ to 7.8$)$ & $\mathrm{p}=0.068$ & $1.9(0.7-3.9)$ & $\mathrm{p}=0.294$ \\
\hline Sepsis & $85(23.5 \%)$ & $31(60.8 \%)$ & $5.1(2.7$ to 9.3$)$ & $\mathrm{p}<0.001$ & $1.6(0.9-3.8)$ & $\mathrm{p}=0.119$ \\
\hline Pneumonia & $46(12.7 \%)$ & $17(33.3 \%)$ & $3.4(1.8$ to 6.6$)$ & $\mathrm{p}<0.001$ & $1.5(0.7-3.0)$ & $\mathrm{p}=0.275$ \\
\hline Central line-associated infections & $25(6.9 \%)$ & $12(23.5 \%)$ & $4.1(1.9$ to 8.9$)$ & $\mathrm{p}<0.001$ & $1.7(0.8-3.9)$ & $\mathrm{p}=0.200$ \\
\hline Urinary tract infections & $15(4.1 \%)$ & $3(5.9 \%)$ & $1.4(0.4$ to 5.2$)$ & $\mathrm{p}=0.57$ & $0.8(0.2-3.2)$ & $\mathrm{p}=0.798$ \\
\hline Wound infection & $12(3.3 \%)$ & $8(15.7 \%)$ & $5.4(2.1$ to 14.0$)$ & $\mathrm{p}<0.001$ & $2.3(0.8-6.2)$ & $\mathrm{p}=0.114$ \\
\hline \multirow[t]{2}{*}{ Mortality } & $48(13.3 \%)$ & $15(29.4 \%)$ & $2.7(1.4$ to 5.4$)$ & $\mathrm{p}=0.004$ & $2.0(0.8-4.9)$ & $\mathrm{p}=0.143$ \\
\hline & & & $\begin{array}{c}\text { unadjusted difference } \\
(95 \% \mathrm{CI})\end{array}$ & & $\begin{array}{l}\text { adjusted difference } \\
(95 \% \mathrm{CI})\end{array}$ & \\
\hline Length of ICU stay (days) & $11(6-25)$ & $33(15-44)$ & $28.0(19.8$ to 36.3$)$ & $\mathrm{p}<0.001$ & $13.7(5.5-21.8)$ & $\mathrm{p}=0.001$ \\
\hline Length of hospital stay (days) & $21(13-35)$ & $39(23-66)$ & $28.7(19.8$ to 37.6$)$ & $\mathrm{p}<0.001$ & $13.5(4.6-22.5)$ & $\mathrm{p}=0.003$ \\
\hline
\end{tabular}

Adjusted for the following confounders: ABSI-score, Charlson co-morbidity index, the presence of additional injuries and administration of clotting factors. All parametric results were presented as median (interquartile range). $\mathrm{OR}=$ odds ratio; $\mathrm{CI}=$ confidence interval; SIRS = Systemic Inflammatory Response Syndrome; 
Table 4: Outcome analysis for PLT transfusions in severely burned patients ( $n=413)$.

\begin{tabular}{|c|c|c|c|c|c|c|}
\hline & $\begin{array}{c}\text { No PLT } \\
\text { transfusions } \\
n=382 \\
\end{array}$ & $\begin{array}{c}\begin{array}{c}\text { Requiring PLT } \\
\text { transfusions } \\
\mathrm{n}=\mathbf{3 1}\end{array} \\
\end{array}$ & unadjusted OR (95\% CI) & p-value & adjusted OR (95\% CI) & p-value \\
\hline Thrombosis & $28(7.3 \%)$ & $7(22.6 \%)$ & $2.4(1.4$ to 4.2$)$ & $\mathrm{p}=0.002$ & $1.9(0.7$ to 5.3$)$ & $\mathrm{p}=0.212$ \\
\hline SIRS & $13(3.4 \%)$ & $6(19.4 \%)$ & $6.8(2.4$ to 19.4$)$ & $\mathrm{p}<0.001$ & $4.5(1.3$ to 15.5$)$ & $\mathrm{p}=0.018$ \\
\hline Sepsis & $93(24.3 \%)$ & $23(74.2 \%)$ & 8.9 (3.8 to 20.6$)$ & $\mathrm{p}<0.001$ & $2.2(0.9$ to 5.5$)$ & $\mathrm{p}=0.101$ \\
\hline Pneumonia & $50(13.1 \%)$ & $13(41.9 \%)$ & $4.8(2.2$ to 10.4$)$ & $\mathrm{p}<0.001$ & $1.9(0.8$ to 4.4$)$ & $\mathrm{p}=0.158$ \\
\hline Central line-associated infections & $30(7.9 \%)$ & $7(22.6 \%)$ & $3.4(1.4$ to 8.6$)$ & $\mathrm{p}=0.009$ & $1.2(0.4$ to 3.4$)$ & $\mathrm{p}=0.706$ \\
\hline Urinary tract infections & $16(4.2 \%)$ & $2(6.5 \%)$ & $1.6(0.3$ to 7.2$)$ & $\mathrm{p}=0.56$ & $0.9(0.2$ to 4.4$)$ & $\mathrm{p}=0.862$ \\
\hline Wound infection & $17(4.5 \%)$ & $3(9.7 \%)$ & $2.3(0.6$ to 8.3$)$ & $\mathrm{p}=0.20$ & $0.6(0.2$ to 2.5$)$ & $\mathrm{p}=0.489$ \\
\hline Mortality & $45(11.8 \%)$ & $18(58.1 \%)$ & 10.4 (4.8 to 22.6$)$ & $\mathrm{p}<0.001$ & $5.8(2.1$ to 16.0$)$ & $\mathrm{p}=0.001$ \\
\hline
\end{tabular}

Adjusted for the following confounders: ABSI-score, Charlson co-morbidity index, the presence of additional injuries and administration of clotting factors. OR $=$ odds ratio; $\mathrm{CI}=$ confidence interval; SIRS = Systemic Inflammatory Response Syndrome; 
Figure 1: Flowchart of patient selection.

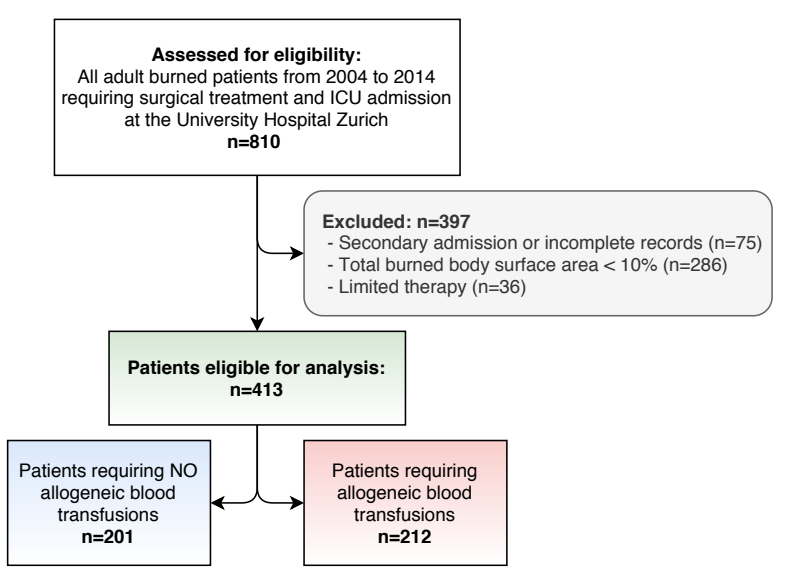

Figure 2: Odds ratios. The effect of allogeneic blood transfusion on clinical outcome in clotting factors.

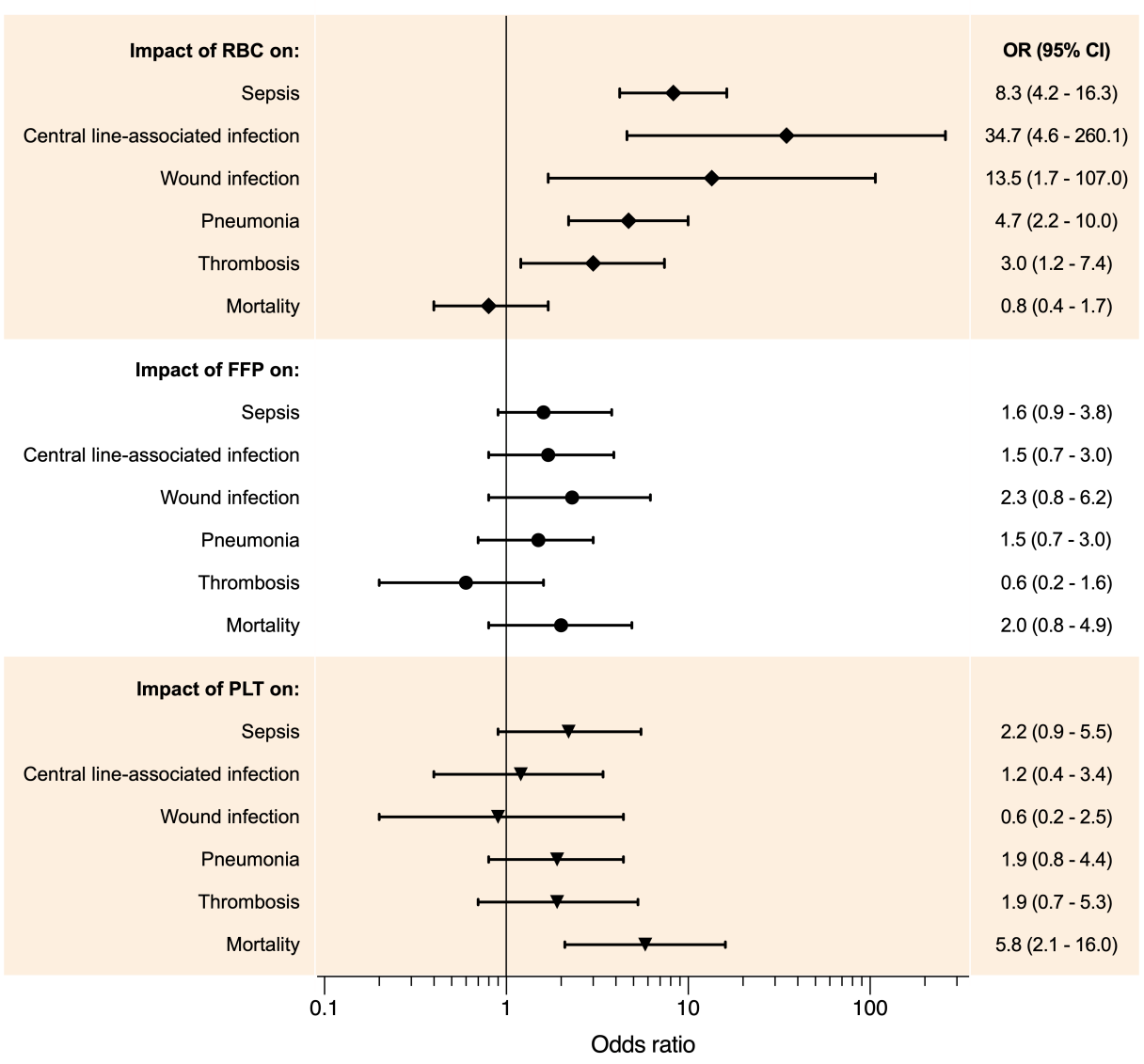

